\title{
MicroRNA-34c-5p Inhibition of NUF2 Suppresses Lung Adenocarcinoma Cell Viability and Invasion
}

\author{
Xiaoguang You, ${ }^{1}$ Haiying Ren, ${ }^{2}$ and Lijun Wen $\mathbb{D D}^{3}$ \\ ${ }^{1}$ The First Affiliated Hospital of Hainan Medical University, China \\ ${ }^{2}$ The Sixth People's Hospital of Jilin City, China \\ ${ }^{3}$ Hainan Medical University, China \\ Correspondence should be addressed to Lijun Wen; wljpd2018@163.com
}

Received 21 May 2021; Revised 1 June 2021; Accepted 3 June 2021; Published 21 June 2021

Academic Editor: Songwen Tan

Copyright ( 2021 Xiaoguang You et al. This is an open access article distributed under the Creative Commons Attribution License, which permits unrestricted use, distribution, and reproduction in any medium, provided the original work is properly cited.

Background. Lung cancer continues to be a burden worldwide with an estimated 2.09 million new cases of lung cancer and 1.76 million deaths in 2018. MicroRNAs (miRs) are key regulators of gene expression and show their oncogenic or antioncogenic role in human cancers including lung cancer. In this study, we test the hypothesis that miR-34c-5p functions as a candidate antioncomiR in lung adenocarcinoma by targeting NUF2. Methods. The expression pattern of miR-34c-5p and NUF2 was evaluated in 202 biopsy specimens from patients with lung adenocarcinoma and 176 biopsy specimens from patients with benign lung diseases. Interaction between miR-34c-5p and NUF2 was verified by the luciferase-based assay. Cell viability and invasion assays were carried out in cultured A549 cells treated with miR-34c-5p mimic, inhibitor, and siRNA against NUF2. Results. NUF2 was highly expressed in lung adenocarcinoma samples and related to the differentiation degree, TNM stage, and presence of lymph node metastasis (LNM). Patients with NUF2 overexpression had reduced overall survival (OS) and diseasefree survival (DFS) compared to patients with underexpression. Cox multivariate analysis revealed that high expression of NUF2, advanced TNM stage, well/moderate differentiation, and existence of LNM were unfavorable prognostic factors. siRNAmediated knockdown of NUF2 inhibits A549 cell viability and invasion. miR-34c-5p was expressed at a poor level in lung adenocarcinoma samples and related to the differentiation degree, TNM stage, and presence of LNM. miR-34c-5p underexpression contributes to reduced OS and DFS, which was demonstrated as an unfavorable prognostic factor by Cox multivariate analysis. siRNA-mediated knockdown of NUF2 could ablate miR-34c-5p inhibition-mediated effects on A549 cells. Conclusion. Our results prove the hypothesis that miR-34c-5p could suppress lung adenocarcinoma progression by binding to the NUF2 gene. The study is a significant step towards extending our understanding of the mode of miRNA regulation in lung adenocarcinoma.

\section{Introduction}

Lung cancer represents the leading cause of death among the population with malignant tumors worldwide [1]. The incidence and mortality of lung cancer in China are increasing rapidly in the past three decades, which imposes a large burden on patients and the society economy [2]. On the basis of annual projections, the National Central Cancer Registry reported that there were approximately 46.08 per 100,000 people suffering from lung cancer and 37.00 per 100,000 people dying of this disease in 2010 [2]. Lung adenocarcinoma is a frequently occurring subtype of lung cancer, originating from the smaller airways and occurring in peripheral lung tissues [3]. However, histopathology is insufficient to monitor disease progression and clinical outcome of patients with lung adenocarcinoma.

Interestingly, lung adenocarcinoma possesses different genomic alterations in contrast to other lung cancer subtypes, which suggests a wide range of molecular variations that underlie the disease [4]. Recently, approaches to lung adenocarcinoma 
treatment advanced from the application of adjuvant chemotherapy to personalized treatment based on genomic alterations [5]. Recently, dysfunctions of microRNAs (miRNAs) are commonly related to lung cancer tumorigenesis by affecting their target genes, which highlights their potential in reducing drug resistance and encouraging histological subclassification techniques [6].

The miR-34 family (miR-34a, miR-34b, and miR-34c) was upregulated in lung cancer, while $\mathrm{miR}-34 \mathrm{~b} / \mathrm{c}$ was demonstrated to be more effective as a tumor suppressor than miR$34 \mathrm{a}$ [7]. Poor expression of miR-34c-5p found in the context of human cancers including colorectal cancer [8] and laryngeal squamous cell carcinoma [9] underlines its antitumor effect. NUF2 (Ndc80 kinetochore complex component) is a molecular linker between tubulin subunits and the kinetochore attachment site at kinetochore-attached microtubule ends [10]. NUF2 has been identified as an oncogene that is overexpressed in the context of several cancers, such as prostate cancer [11], pancreatic cancer [12], and colorectal cancers [13]. Increasing bioinformatics prediction shows that miR$34 c-5 p$ has a chance to interact with NUF2 $3^{\prime}$-UTR. Therefore, we determined the expression level of miR-34c-5p and NUF2 in bronchial biopsy samples from patients with lung adenocarcinoma and benign lung diseases and correlate it with clinical features and prognosis. Furthermore, we manipulated miR-34c-5p expression in lung adenocarcinoma cell A549 and assess its effect on cell viability and invasion.

\section{Materials and Methods}

2.1. Gene Expression Profiles of NPC in Gene Expression Omnibus (GEO). Microarray data deposited with GEO (https://www.ncbi.nlm.nih.gov/geo/) under accession number GSE33532 between individual primary tumors and matched distant normal lung tissues were analyzed. An absolute $\mid \log 2$ fold change (FC) $\mid>0.5$ plus a false discovery rate (FDR) $p<0.05$ was used to screen as differentially expressed genes (DEGs) using the Limma package of $\mathrm{R}$ language between individual primary tumors and matched distant normal lung tissues.

2.2. Tissue Specimen Collection. Two hundred and two biopsy specimens were based on patients pathologically diagnosed with lung adenocarcinoma and undergoing bronchoscopy at our hospital from January 2009 to December 2015. The mean age of enrolled patients was $61.62 \pm 4.34$ years, and none of them received chemotherapy or radiotherapy. The tumor node metastasis (TNM) of 202 specimens was split into different stages according to the staging criteria formulated by the International Association for the Study of Lung Cancer (IASLC) [14]. The follow-up study was performed from January 2009 to December 2020, with overall survival (OS) and disease-free survival (DFS) recorded. During the same period, 176 biopsy specimens were collected from patients with benign lung diseases who visited our hospital, and there were 103 males and 73 females, with a mean age of $58.92 \pm 5.23$ years.
2.3. Immunohistochemistry (IHC). Tissues were fixed with $10 \%$ formaldehyde, paraffin-embedded, and cut into sections $(4 \mu \mathrm{m})$. Sections were stained using anti-NUF2 (Santa Cruz Biotechnology, USA) and then allowed to react with goat anti-rabbit IgG (Beyotime Institute of Biotechnology, China) for $30 \mathrm{~min}$. We randomly captured 5 fields of view under 200x magnification using a microscope (Nikon, Japan). Extents of staining were reflected using scores: 0 indicates no stained cells, 1 indicates $1-25 \%$ stained cells, 2 indicates $26-$ $50 \%$ stained cells, and 3 indicates $51-100 \%$ stained cells. With regard to staining intensities, 0 reflects uncolored or not obvious stain, 1 reflects light yellow stains, 2 reflects brownish yellow stains, and 3 reflects brown stains. Final staining scores consisted of intensity and extent scores. The score was 0-1 point as negative (-), 2-3 points as weakly positive $(+)$, and 4-6 points as positive (++). In this study, both “-” and "+" were regarded as negative and " ++ " as positive.

2.4. Manipulation of miR-34c-5p and NUF2 In Vitro. A549 cells were harvested in RPMI-1640 with $10 \%$ fetal bovine serum (FBS) at $37^{\circ} \mathrm{C}$ under $5 \% \mathrm{CO}_{2}$. Once cell confluence reached $70 \%-90 \%$, the old medium was renewed with serum-free medium $(1600 \mu \mathrm{L}) . \mathrm{miR}-34 \mathrm{c}-5 \mathrm{p}$ mimic, control mimic, miR-34c-5p inhibitor, control inhibitor, siRNA targeting NUF2, and scramble siRNA (GenePharma, China) were separately delivered into A549 cells ( $400 \mu \mathrm{L}$ supplemented with Lipofectamine 2000) as per the manufacturer's protocols (Invitrogen, USA). Transient transfection lasted for 48 hours.

2.5. Real-Time qPCR (RT-qPCR). Total RNA was obtained by the method of TRIzol (No. 15596026, Invitrogen, USA). The cDNA was generated using PrimeScript ${ }^{\mathrm{TM}}$ II 1st Strand cDNA Synthesis Kits (TaKaRa) for mRNA quantification. RT-qPCR was completed as instructed by the manual provided by the kit (SYBR Premix Ex Taq Kit, TaKaRa) on the real-time PCR system (Applied Biosystems, USA). The primer sequences of NUF2 were $5^{\prime}$-CACACCAGGAGGCATT AATGAAACT-3' (forward) and $5^{\prime}$-GGAATTTCCCTCTT GCAGCACT- $3^{\prime}$ (reverse), and the primer sequences of GAPDH were $5^{\prime}$-ATGGGGAAGGTGAAGGTCGG-3 ${ }^{\prime}$ (forward) and $5^{\prime}$-GACGGTGCCATGGAATTTGC-3' (reverse). The cDNA was generated using the One Step PrimeScript miRNA cDNA Synthesis Kit (TaKaRa, Japan) for miRNA quantification. The primer sequence of hsa-miR-34c-5p was $5^{\prime}$-AGGCAGTGTAGTTAGCTGATTGC-3', and the primer sequence of U6 was $5^{\prime}$-CGCAAGGATGACACGC AAATTC- $3^{\prime}$. The expression of miR-34c-5p was relative to U6 snRNA and NUF2 to GAPDH.

2.6. Western Blotting. After 10\% SDS-PAGE followed by membrane transfer, Western blots were probed with antiNUF2 (Santa Cruz Biotechnology) and incubation with goat anti-rabbit IgG (Beyotime Institute of Biotechnology). GAPDH was used as a loading control for normalization. Visualization for Western blots was performed by means of an enhanced chemiluminescence (ECL) method (Amersham Pharmacia, USA), and gray quantification of Western blots was analyzed using ImageJ v1.48u software. 


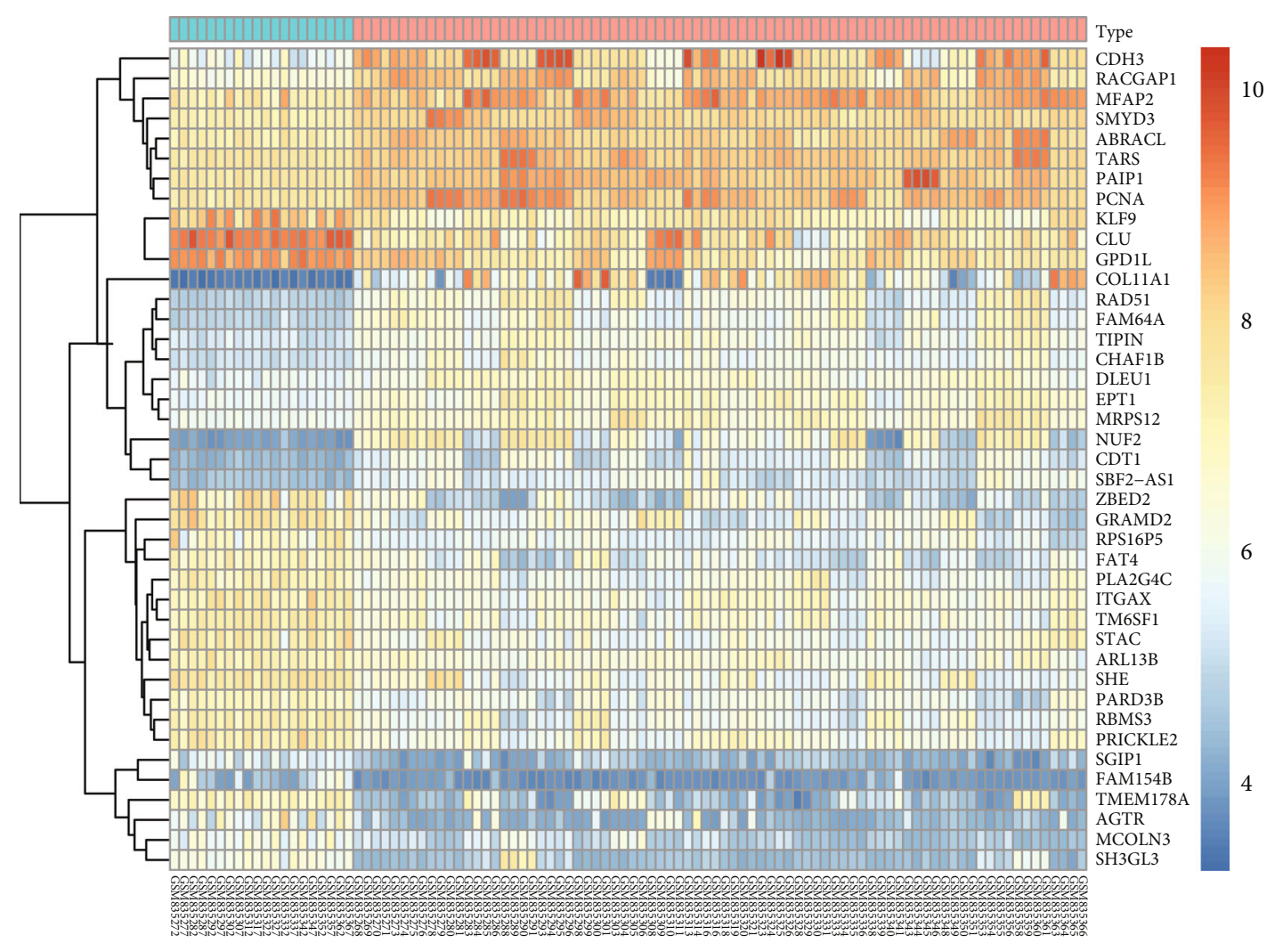

Type

Control

NSCLC

(a)
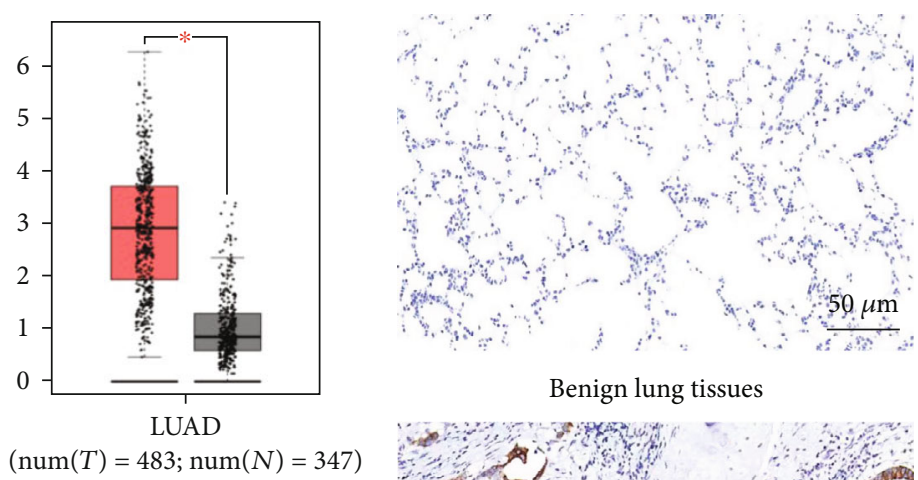

Benign lung tissues

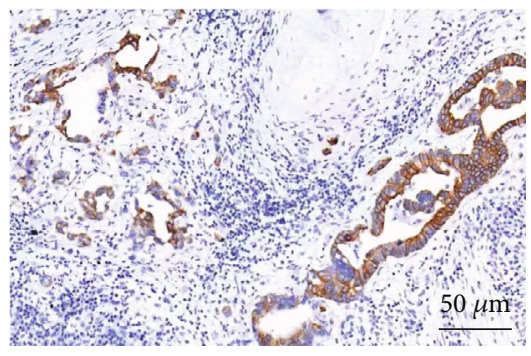

Lung adenocarcinoma tissues

(b)

(c)

FIgURe 1: Continued. 


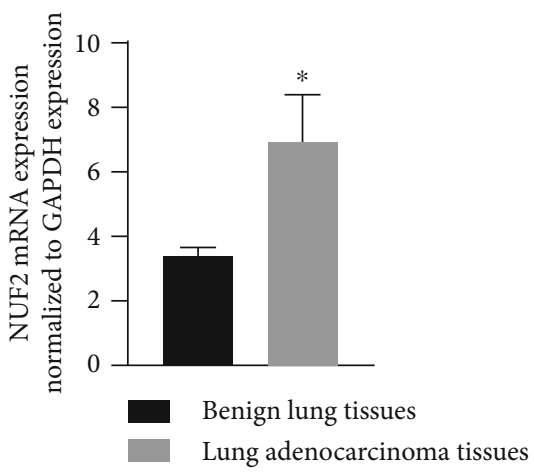

(d)

FIGURE 1: NUF2 is overexpressed in lung adenocarcinoma tissues. (a) DEGs between primary tumors $(n=20)$ and matched distant normal lung tissues $(n=20)$ in the GSE33532 dataset are shown in a heat map. Color scale indicates degrees of downregulation (green) and upregulation (red). (b) The expression of NUF2 in primary lung tumors $(n=483)$ and normal lung tissues $(n=347)$ in the GEPIA database. (c) Representative image of immunohistochemical staining for NUF2 in lung adenocarcinoma tissues $(n=202)$ and negative expression of NUF2 in benign lung tissues $(n=176)$; original magnification, $\times 200$. (d) The mRNA expression of NUF2 in lung adenocarcinoma tissues $(n=202)$ and benign lung tissues $(n=176)$ determined by RT-qPCR. ${ }^{*} p<0.05$.

2.7. Luciferase Activity Assays. The wild-type NUF2 and NUF2 mutated at the putative binding sites of miR-34c-5p were independently inserted into luciferase reporter plasmids (Promega, USA), named pmirGLO-NUF2-Wt and pmirGLO-NUF2Mut. HEK293T cells were cotransfected with reporter plasmids and either miR-34c-5p mimic or control mimic using the dual-luciferase reporter assay system (D0010, Solarbio, China).

2.8. Cell Viability and Invasion Assays. Cell viability was reflected by absorbance at different incubation with $4 \mathrm{~h}$ incubation of $10 \mu \mathrm{L}$ MTT, with growth curves plotted accordingly. When performing cell invasion assays, the cells were resuspended into $3 \times 10^{5}$ cells per well using serum-free RPMI1640 medium and then added to the upper chambers (BD Biosciences, USA) coated with Matrigel (BD Biosciences). Matrigel was pretreated using serum-free DMEM $(1: 10)$. The lower chamber was filled with DMEM with 5\% FBS as for chemoattractant. Twenty-four hours later, the cells stained with $0.1 \%$ crystal violet in the lower chamber were counted in six fields with a microscope (Olympus, Tokyo, Japan).

2.9. Data Processing. All statistics were performed using SPSS Statistics 21.0. Data were displayed as the percentage or mean \pm standard deviation and compared using the chisquared test or unpaired $t$-test between two groups, with $p$ $<0.05$ regarded as a significant difference. Survival probabilities were estimated by the Kaplan-Meier method and logrank test. Cox multivariate regression analysis was employed to identify independent risk factors for patient survival.

\section{Results}

3.1. Overexpression of NUF2 in Lung Adenocarcinoma Tissues. After analyzing raw data from the GSE33532 dataset, 50 DEGs between lung cancer tissue samples than their normal counterparts stood out. We depicted a heat map (Figure 1(a)) showing 50 DEGs between primary tumors and matched
TABLE 1: NUF2 expression was related to differentiation, TNM stage, and the presence of LNM in lung adenocarcinoma.

\begin{tabular}{|c|c|c|c|c|}
\hline Pathological index & Case & $\begin{array}{l}\text { Positive rate of } \\
\text { NUF2, } n(\%)\end{array}$ & $\chi^{2}$ & $p$ \\
\hline \multicolumn{5}{|l|}{ Gender } \\
\hline Male & 113 & $63(55.75)$ & \multirow{2}{*}{0.363} & \multirow{2}{*}{0.717} \\
\hline Female & 89 & $54(60.67)$ & & \\
\hline \multicolumn{5}{|l|}{ Age } \\
\hline$<60$ & 63 & $35(55.56)$ & \multirow{2}{*}{0.238} & \multirow{2}{*}{0.812} \\
\hline$\geq 60$ & 139 & $82(58.99)$ & & \\
\hline \multicolumn{5}{|l|}{ Smoking history } \\
\hline Yes & 71 & $43(60.53)$ & \multirow{2}{*}{0.475} & \multirow{2}{*}{0.635} \\
\hline No & 131 & $74(56.49)$ & & \\
\hline \multicolumn{5}{|c|}{ Maximal tumor diameter } \\
\hline$<3 \mathrm{~cm}$ & 143 & $79(55.24)$ & \multirow{2}{*}{0.612} & \multirow{2}{*}{0.541} \\
\hline$\geq 3 \mathrm{~cm}$ & 59 & $38(64.41)$ & & \\
\hline \multicolumn{5}{|l|}{ TNM stage } \\
\hline $\mathrm{I}+\mathrm{II}$ & 121 & $52(42.98)$ & \multirow{2}{*}{2.670} & \multirow{2}{*}{0.008} \\
\hline $\mathrm{III}+\mathrm{IV}$ & 81 & $65(80.25)$ & & \\
\hline \multicolumn{5}{|l|}{ Differentiation } \\
\hline $\begin{array}{l}\text { Medium/well } \\
\text { differentiation }\end{array}$ & 166 & $84(50.60)$ & \multirow[t]{2}{*}{2.045} & \multirow[t]{2}{*}{0.030} \\
\hline Poor differentiation & 36 & $33(91.67)$ & & \\
\hline \multicolumn{5}{|l|}{ LNM } \\
\hline Presence & 60 & $50(83.33)$ & \multirow{2}{*}{2.360} & \multirow{2}{*}{0.018} \\
\hline Absence & 142 & $67(47.18)$ & & \\
\hline
\end{tabular}

distant normal lung tissues from the GSE33532 dataset. The GEPIA database also showed higher expression of NUF2 in cancer samples than their normal counterparts in lung adenocarcinoma (Figure 1(b)). However, NUF2 has been 

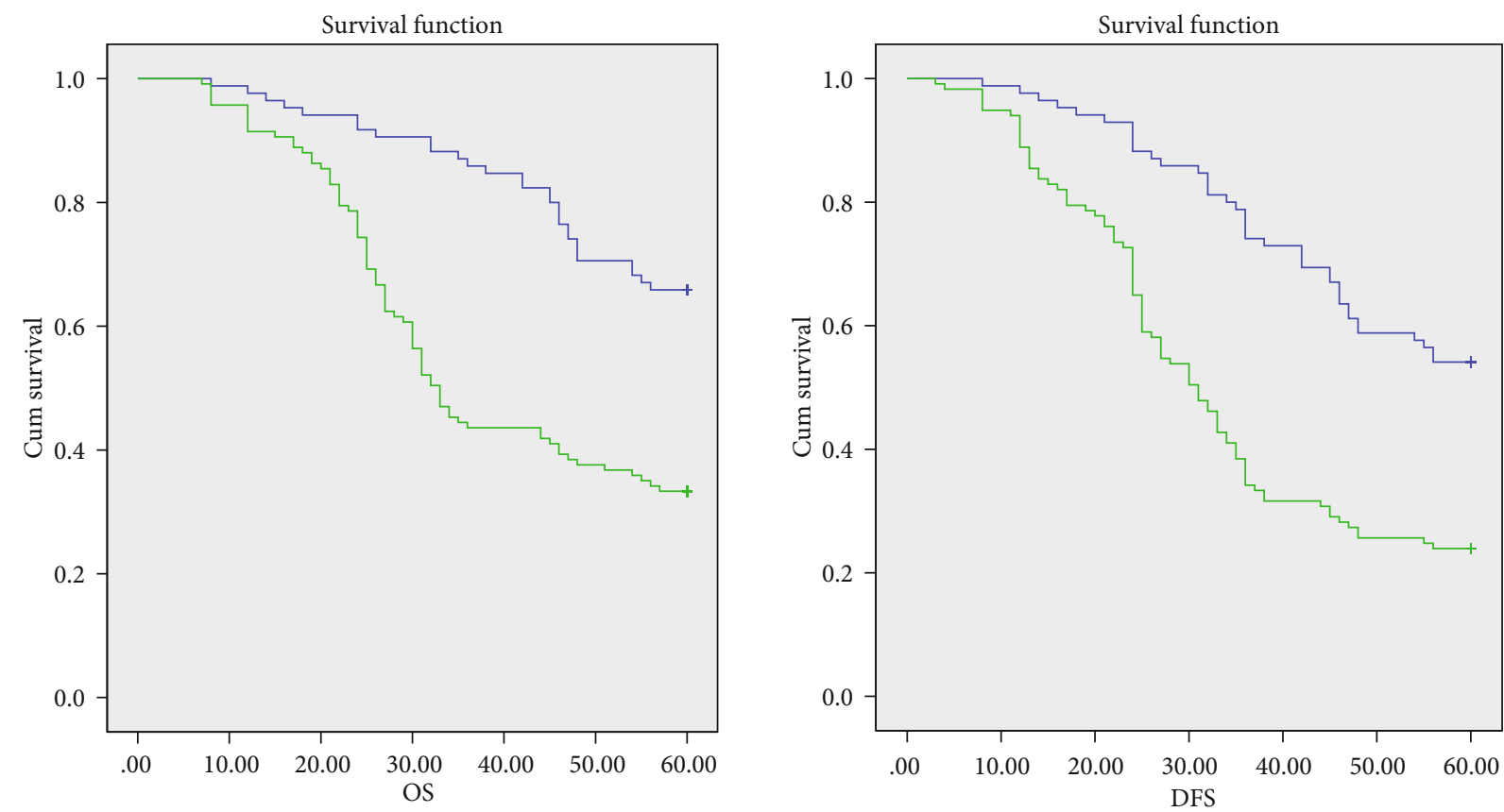

$$
\begin{aligned}
& \text { NUF2 } \\
& \neg \text { Negative } \\
& -\neg \text { Positive } \\
& + \text { Negative censored } \\
& + \text { Positive censored }
\end{aligned}
$$

Figure 2: Kaplan-Meier curves were depicted to estimate OS and DFS of a patient with lung adenocarcinoma $(n=202)$ according to NUF2 expression. The median OS and DFS time for the positive expression group was 38.6 months and 34.2 months, respectively. The median OS and DFS time for the negative group was 52.3 months and 48.7 months, respectively.

TABLE 2: Multivariate survival analysis in Cox proportional hazards models for prognosis of lung adenocarcinoma.

\begin{tabular}{lcccccr}
\hline Factors & \multirow{2}{*}{ S } & \multirow{2}{*}{ SE } & Wald & SIG. & Exp.(B) & \multicolumn{2}{c}{ 95\% CI for Exp.(B) } \\
Lower & Upper \\
\hline TNM & 0.793 & 0.268 & 8.742 & 0.003 & 2.210 & 1.306 \\
Differentiation & -0.497 & 0.252 & 3.893 & 0.048 & 0.609 & 0.372 \\
LNM & 0.559 & 0.275 & 4.121 & 0.042 & 1.749 & 1.019 \\
High expression of NUF2 & 0.664 & 0.243 & 7.454 & 0.006 & 1.943 & 3.001 \\
\hline
\end{tabular}

investigated rarely in lung adenocarcinoma in recent studies. In order to further confirm the expression patterns of NUF2 in lung adenocarcinoma, we collected 202 biopsy specimens from lung adenocarcinoma patients and 176 biopsy specimens from patients with benign lung diseases. Immunohistochemical staining for NUF2 was performed to estimate the positive expression of NUF2 between lung adenocarcinoma tissues and benign lung tissues. It was found that NUF2 was mainly localized in the cytoplasm as yellow-brown particles. Among 202 lung adenocarcinoma tissues, the positive rate of NUF2 reached 57.92\% (117/202). Among 176 benign lung tissues, no positive staining for NUF2 was observed. The positive rate of NUF2 was notably higher in lung adenocarcinoma tissues than in benign lung tissues $(p<0.05$, Figure $1(\mathrm{c}))$. RT-qPCR showed that lung adenocarcinoma tissues exhibited a higher
mRNA expression of NUF2 than benign lung tissues $(p<0.05$, Figure $1(\mathrm{~d}))$.

3.2. Overexpression of NUF2 Contributes to Lung Adenocarcinoma Progression. Since NUF2 overexpression was observed in lung adenocarcinoma tissues, the focus of our analysis was shifted to studying the relationship between NUF2 activity and clinical variables of lung adenocarcinoma patients. We collected 202 biopsy specimens from lung adenocarcinoma patients and 176 biopsy specimens from patients with benign lung diseases, with medical records. We found that NUF2 expression was related to differentiation, TNM stage, and the existence of lymph node metastasis (LNM), as evidenced by the results that patients in stage III and stage IV, with poor differentiation, and with LNM 


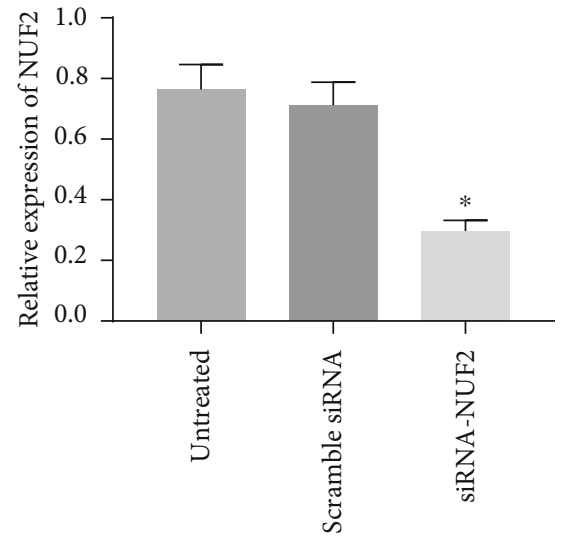

(a)

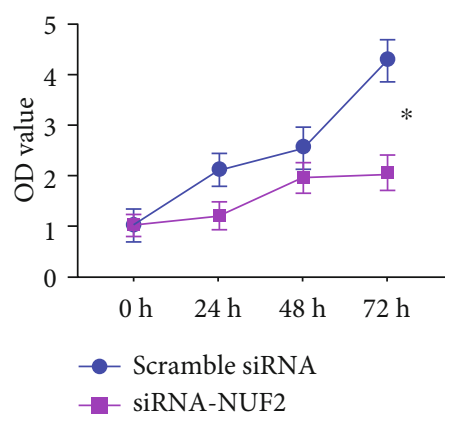

(c)

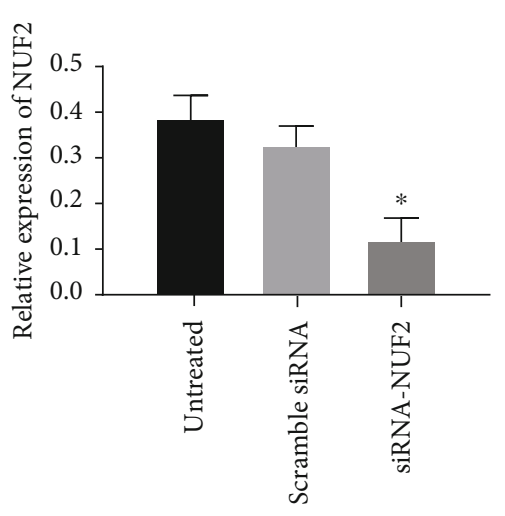

(b)

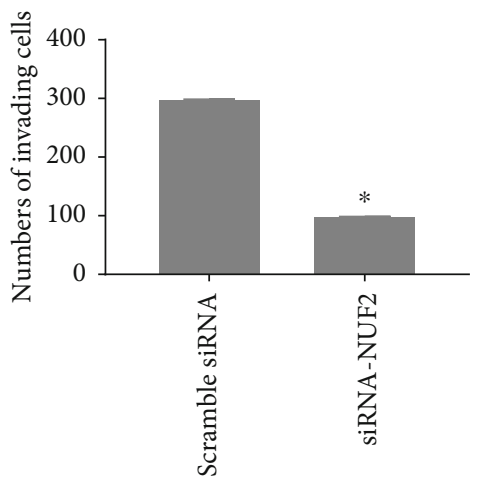

(d)

FIgURe 3: Knockdown of NUF2 inhibits lung adenocarcinoma A549 cell viability and invasion. (a, b) siRNA targeting NUF2 was delivered into A549 cells to specifically blunt NUF2 expression in vitro, with delivery of scramble siRNA as the negative control. NUF2 mRNA and protein expressions were assessed in A549 cells by the RT-qPCR and Western blot analysis. (c) Cell viability was examined by MTT assays. (d) Cell invasion was examined by Transwell assays. ${ }^{*} p<0.05$.

presented a higher positive rate of NUF2 than those in stage I and stage II, with well/moderate differentiation, and without LNM ( $p<0.05$, Table 1). Collectively, NUF2 overexpression may be associated with lung adenocarcinoma progression.

3.3. NUF2 Overexpression Predicted Poor Survival of Lung Adenocarcinoma. TCGA also showed that there was a significant difference concerning lung adenocarcinoma OS between patients with high and low expressions of NUF2 $(p=0.0025)$. The intensity and extent of NUF2 staining were evaluated, and two scores were added. Patients with - and + were classified into the negative expression group and those with ++ into the positive expression group. Patients with NUF2 overexpression had reduced OS and DFS compared to patients with underexpression $(p<0.05$, Figure 2$)$. Next, we included high expression of NUF2, TNM stage, differentiation, and LNM into the Cox proportional hazards model for the prognosis of lung adenocarcinoma. The results of Cox multivariate analysis revealed that (Table 2) high expression of NUF2, TNM stage, differentiation, and LNM were independent risk factors for the prognosis of lung adenocarcinoma $(p<0.05)$.

3.4. Knockdown of NUF2 Inhibits Lung Adenocarcinoma A549 Cell Viability and Invasion. We manipulated NUF2 using siRNA in A549 cells and examined the effect of NUF2 on A549 cell viability and invasion. First, the RTqPCR analysis and Western blotting determined that siRNA designed specifically for NUF2 successfully knocked down the mRNA expression of NUF2 and diminished the protein level of NUF2 (Figures 3(a) and 3(b)). The results of MTT assays and Transwell invasion assays revealed that compared with A549 cells treated with scramble siRNA, siRNA-NUF2treated A549 cells showed reduced cell viability and invasion $(p<0.05$, Figures 3(c) and 3(d)).

3.5. Downregulated miR-34c-5p Was Associated with the Development and Poor Prognosis of Lung Adenocarcinoma. To further evaluate the expression pattern of miR-34c-5p in lung adenocarcinoma, we quantified miR-34c-5p in lung adenocarcinoma tissues and benign lung tissues. As shown in Table 3, patients in stage III and stage IV, with poor differentiation, and with lymph node metastasis (LNM) presented a lower expression level of miR-34c-5p than those in stage I and stage II, with well/moderate differentiation, and without LNM $(p<0.05)$. Subsequently, Kaplan-Meier curves were depicted to estimate patient OS and DFS according to miR$34 c-5 p$ expression $(p<0.05)$. The results showed that 
TABle 3: Association between miR-34c-5p expression and clinical variables of lung adenocarcinoma patients.

\begin{tabular}{|c|c|c|c|}
\hline Index & $\begin{array}{l}\text { The expression of miR- } \\
34 c-5 p\end{array}$ & $t$ & $p$ \\
\hline \multicolumn{4}{|l|}{ Gender } \\
\hline Male & $0.59 \pm 0.16$ & \multirow{2}{*}{0947} & \multirow{2}{*}{0.345} \\
\hline Female & $0.57 \pm 0.0 .14$ & & \\
\hline \multicolumn{4}{|l|}{ Age } \\
\hline$<60$ & $0.57 \pm 0.21$ & \multirow{2}{*}{0.959} & \multirow{2}{*}{0.588} \\
\hline$\geq 60$ & $0.59 \pm 0.15$ & & \\
\hline \multicolumn{4}{|l|}{ Smoking history } \\
\hline Yes & $0.60 \pm 0.16$ & \multirow{2}{*}{0.996} & \multirow{2}{*}{0.321} \\
\hline No & $0.57 \pm 0.15$ & & \\
\hline \multicolumn{4}{|c|}{ Maximal tumor diameter } \\
\hline$<3 \mathrm{~cm}$ & $0.59 \pm 0.15$ & \multirow{2}{*}{1.383} & \multirow{2}{*}{0.168} \\
\hline$\geq 3 \mathrm{~cm}$ & $0.56 \pm 0.15$ & & \\
\hline \multicolumn{4}{|l|}{ TNM stage } \\
\hline $\mathrm{I}+\mathrm{II}$ & $0.61 \pm 0.16$ & \multirow{2}{*}{3.383} & \multirow{2}{*}{0.001} \\
\hline III+IV & $0.54 \pm 0.13$ & & \\
\hline \multicolumn{4}{|l|}{ Differentiation } \\
\hline $\begin{array}{l}\text { Medium/well } \\
\text { differentiation }\end{array}$ & $0.64 \pm 0.18$ & \multirow[t]{2}{*}{2.581} & \multirow[t]{2}{*}{0.011} \\
\hline Poor differentiation & $0.57 \pm 0.14$ & & \\
\hline \multicolumn{4}{|l|}{ LNM } \\
\hline Presence & $0.54 \pm 0.14$ & \multirow{2}{*}{2.256} & \multirow{2}{*}{0.025} \\
\hline Absence & $0.60 \pm 0.15$ & & \\
\hline
\end{tabular}

patients with low miR-34c-5p expression exhibited poor OS and DFS when compared to those with high miR-34c-5p expression (Figure 4). Next, we included low expression of miR-34c-5p, TNM stage, differentiation, and LNM into the Cox proportional hazards model for the prognosis of lung adenocarcinoma. After Cox multivariate analysis, it was revealed that miR-34c-5p underexpression, TNM stage, differentiation, and LNM were independent risk factors for lung adenocarcinoma prognosis $(p<0.05$, Table 4$)$.

3.6. miR-34c-5p Restrained A549 Cell Viability and Invasion by Targeting NUF2. In this part, we aim to prove the hypothesis that the anticarcinogenic effect of miR-34c-5p on lung adenocarcinoma is achieved by targeting NUF2. At first, we searched web-available databases RNA22 where miR-34c-5p sharing binding sites with NUF2 are predicted (Figure 5(a)) and starBase (http://starbase.sysu.edu.cn/) where miR-34c-5p and NUF2 are coexpressed in lung adenocarcinoma (Figure 5(b)). After dual-luciferase reporter gene assays, it was found that the pmirGLO-NUF2-WT exhibited reduced luciferase intensity upon miR-34c-5p mimic transfection $(p<0.05$, Figure 5(c)). The qRT-PCR and Western blot analysis $(p<0.05$, Figure $5(\mathrm{~d}))$ demonstrated that NUF2 was decreased in A549 cells treated with miR-34c-3p mimic and increased in A549 cells treated with miR-34c-3p inhibitor at mRNA and protein levels. Finally, A549 cells were treated with miR-34c-3p inhibitor and siRNA-NUF2, and their viability and invasion were evaluated using MTT assays and Transwell invasion assays. When compared to A549 cells treated with NC inhibitor followed by scrambled siRNA, A549 cells treated with miR-34c-3p inhibitor followed by siRNA-NUF2 did not differ concerning cell viability and invasion $(p<0.05$, Figures 5(e) and 5(f)), suggesting that miR-34c-3p mediated A549 cell viability and invasion by binding to NUF2.

\section{Discussion}

Lung adenocarcinoma, the most frequent histological subtype of non-small-cell lung cancer (NSCLC), has been indicated to be closely correlated with molecular heterogeneity [4]. Recently, the significance of miRNAs in lung cancer has been increasingly recognized regarding their therapeutic and diagnostic values [15]. In the current study, we synthesized the evidence of miR-34c-5p as a prognostic biomarker in lung adenocarcinoma and provided available evidence validating the relationship between poorly expressed miR-34c$5 \mathrm{p}$ and unfavorable oncologic outcomes of patients with lung adenocarcinoma. Furthermore, elevated miR-34c-5p was observed to exert inhibitory effects on lung adenocarcinoma cell viability and invasion by inhibiting NUF2.

miR-34c-5p was confirmed to be poorly expressed in lung adenocarcinoma in association with poor prognosis, setting a foundation for subsequent experiments. Importantly, miR-34c-5p has been acknowledged to be of great importance in lung-related diseases $[16,17]$. Besides, miR$34 c-5 p$ has been suggested as a target for potential therapeutic strategies in cancer pain management [18]. The association between miR-34c-5p and overall survival condition of patients with lung adenocarcinoma has also been elucidated, which is largely in agreement with our finding [19]. In addition, the prognostic value of miR-34c-5p at the low expression level is consistent with its role in colon cancer according to a previous report [20]. Likewise, miR-34c-5p underexpression has been defined as an independent factor of dismal overall and DFS in patients with laryngeal squamous cell carcinoma [9]. Dysregulation of miRNAs has been identified to affect downstream targets and further function as regulators of various tumorigenic events during the progression and development of cancer [21]. When miR-34c$5 p$ expression was forced in our study, A549 cell abilities of migration and invasion were significantly weakened. A functional study has unraveled the antiapoptotic effects of miR$34 c-5 p$ in A549 cells induced by paclitaxel in lung cancer [22]. Similarly, upregulation of miR-34c-5p has been explored to be involved in the protection mechanism of Pien Tze Huang in colorectal cancer [23]. In the context of cervical cancer, overexpressed miR-34c-5p has the ability to curtail cancer cell proliferation and migration [24], supporting our validation on the involvement of miR-34c-5p in lung adenocarcinoma.

It has been underlined that the integration of miRNAs and target genes may manifest itself as a promising approach 

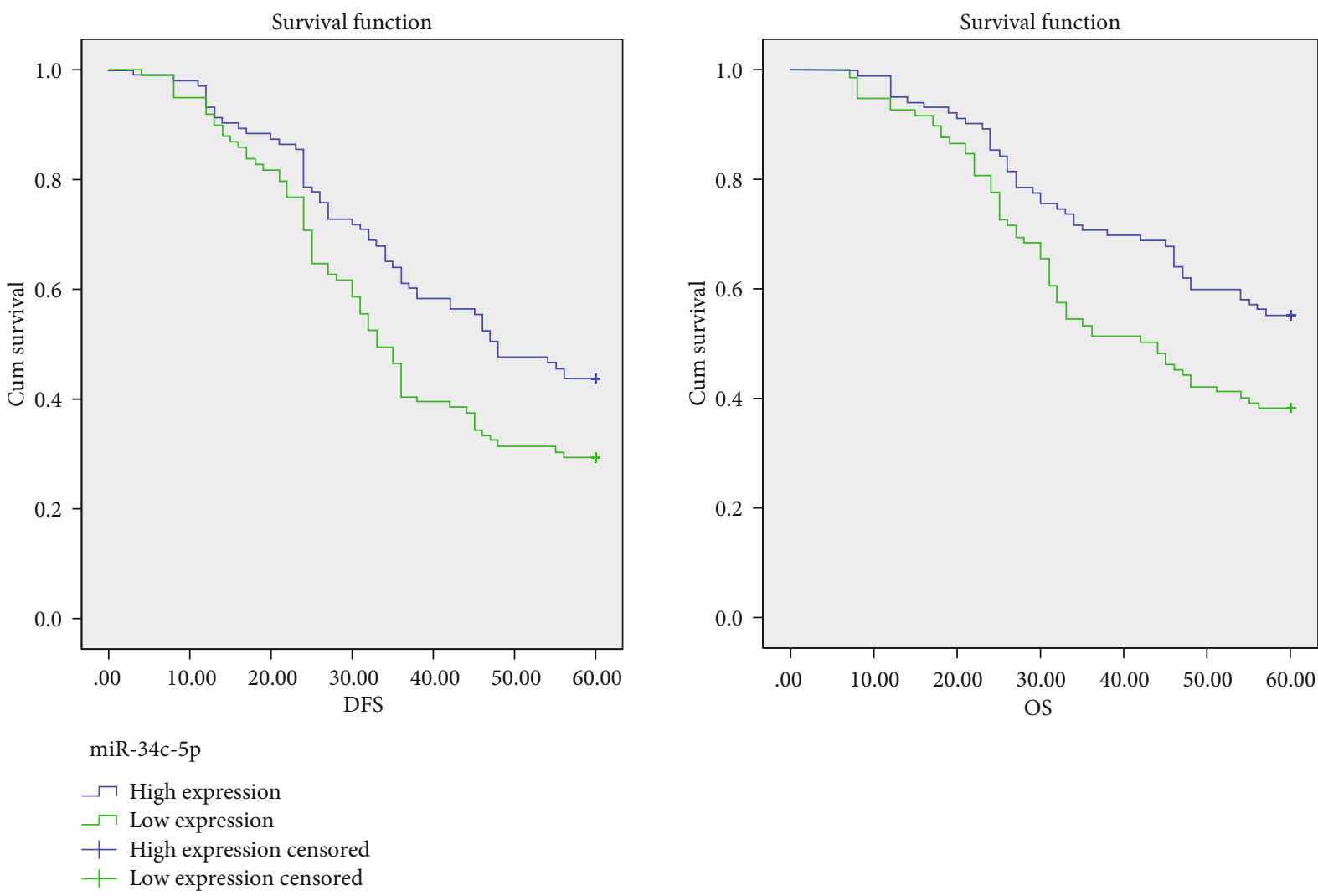

FIgURE 4: Kaplan-Meier curves were depicted to estimate lung adenocarcinoma patient $(n=202)$ OS and DFS according to miR-34c-5p expression. The median OS and DFS time for the low miR-34c-5p group was 44 months and 33.0 months, respectively. The median OS and DFS time for the high miR-34c-5p group was 54.0 months and 48.0 months, respectively.

TABLE 4: Independent risk factors for prognosis of lung adenocarcinoma.

\begin{tabular}{lcccccr}
\hline Factors & $B$ & SE & Wald & SIG. & Exp.(B) & \multicolumn{2}{c}{$\begin{array}{c}\text { 95\% CI for Exp.(B) } \\
\text { Lower }\end{array}$} \\
\hline TNM & 0.570 & 0.234 & 5.925 & 0.015 & 1.768 & 1.117 \\
Differentiation & 0.287 & 0.101 & 8.021 & 0.005 & 1.332 & 1.092 \\
LNM & 0.497 & 0.244 & 4.164 & 0.041 & 1.645 & 1.020 \\
miR-34c-5p & 0.409 & 0.202 & 3.978 & 0.046 & 1.496 & 1.624 \\
\hline
\end{tabular}

for the prediction and progression of NSCLC [25]. During the investigation, it was found that miR-34c-5p could target and further regulate NUF2 in a negative manner, which was considered the underlying mechanism responsible for the antitumor potential of miR-34c-5p against lung adenocarcinoma. Similar interplay involving miR-34c-5p has been elaborated that special AT-rich sequence-binding protein 2 is a specific target of miR-34c-5p in colorectal cancer and sirtuin 6 works in tandem with miR-34c-5p in regulating colon cancer progression $[8,26]$. NUF2, a key mediator of meiotic cell cycle distribution in mammalian oocytes, harbors the property to give rise to defective spindles, activated spindle assembly checkpoint, and misaligned chromosomes [27]. Notably, NUF2 has been documented to play an important part in cell senescence after irradiation, which may result in serious deadly lung injury featured with respiratory failure and pulmonary dysfunction [28]. Mounting evidences have emerged regarding NUF2-targeted therapies to combat multiple cancers, including breast cancer, hepatocellular carcinoma, and pancreatic cancer [29-31]. In lung cancer, NUF2 has also been found to be engaged by interacting with ZW10 interacting protein, a critical part of the mitotic checkpoint with prognostic value [32]. Additionally, NUF2 can be targeted by miR-3613-3p and highlighted as a potential biomarker of liver cancer [33]. Given the aforementioned data and studies, we were convinced that miR-34c-5p-dependent NUF2 inhibition conferred protection against cancerigenic behaviors of lung adenocarcinoma cells.

To sum up, our findings demonstrated that the target inhibition of miR-34c-5p on NUF2 imparted a pronounced inhibitory effect on malignant cell viability and invasion in lung adenocarcinoma, shedding light on the remarkable 


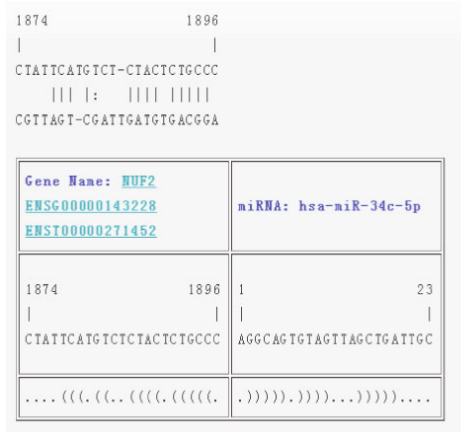

(a)

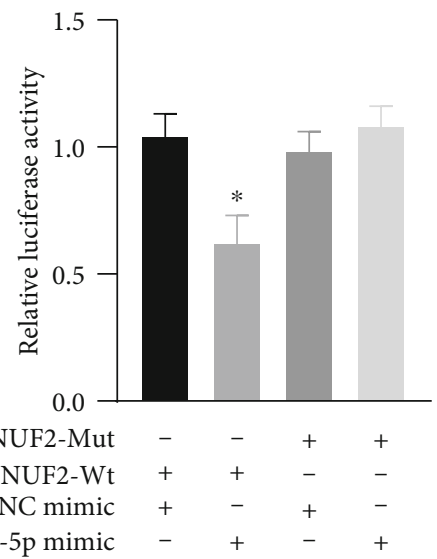

(c)

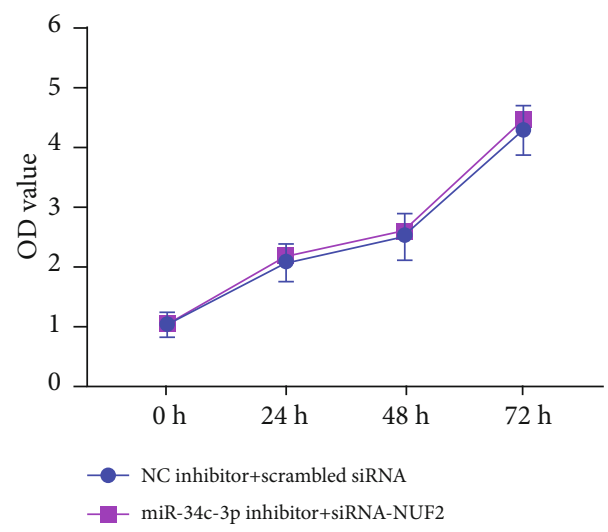

(e)

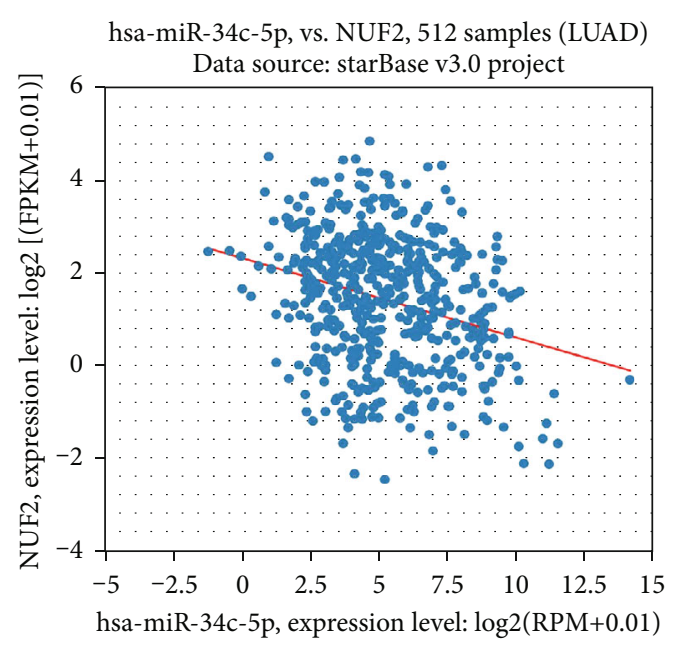

- Regression $(y=-0.1717 x+2.1064)$

$r=-0.278, p^{- \text {value }}=1.58 \mathrm{e}^{-10}$

(b)
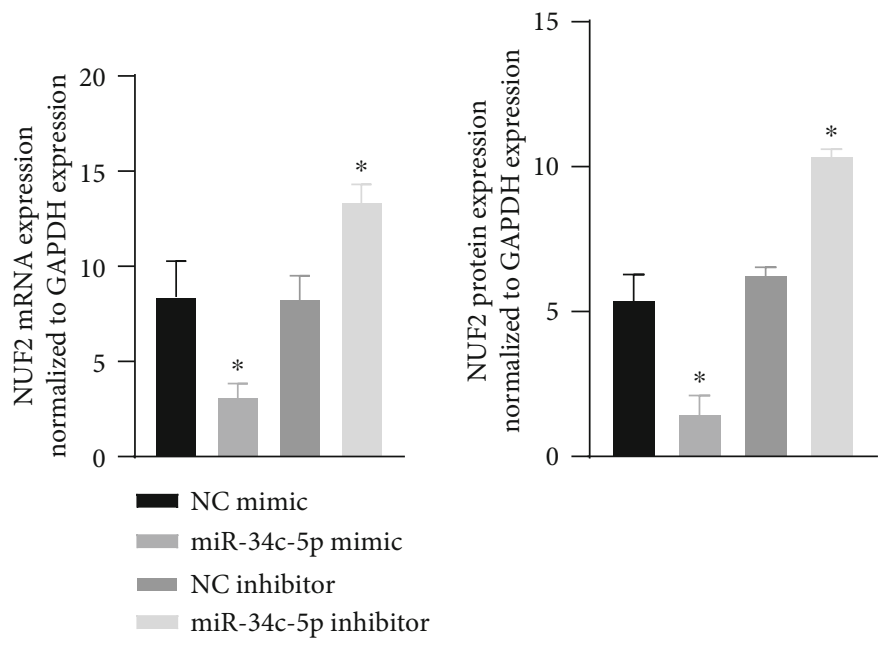

(d)

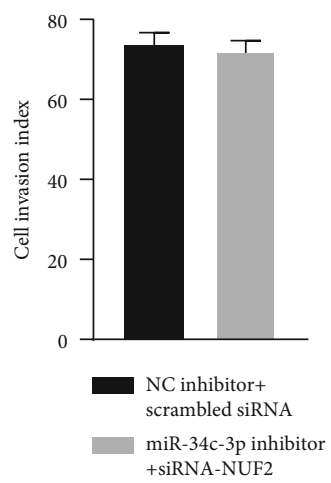

(f)

Figure 5: miR-34c-3p mediates A549 cells by binding to NUF2. (a) The binding sites of miR-34c-5p and NUF2 are predicted in the RNA22 database (https://cm.jefferson.edu/). (b) Coexpression of miR-34c-5p and NUF2 in lung adenocarcinoma in the starBase database (http:// starbase.sysu.edu.cn/). (c) NUF2 as the target gene of miR-34c-3p was verified by luciferase activity assays $(*$ stands for $p<0.05$ when compared with pmirGLO-NUF2-MUT+miR-34c-5p mimic). (d) NUF2 mRNA and protein expressions in A549 cells were determined by qRT-PCR and Western blot analysis, normalized to GAPDH ( $*$ stands for $p<0.05$ when compared with NC mimic or NC inhibitor). (e) Cell viability was examined by MTT assays. (f) Cell invasion was examined by Transwell assays. 
potential of miR-34c-5p as a prognostic biomarker for lung adenocarcinoma. However, further investigations with regard to their functional roles in vivo are expected to make inroads into the implication of miRNA-mRNA in the treatment and prognosis of lung cancer. Besides, more in-depth researches as well as combined research efforts are needed to validate its applicable value in clinical practice.

\section{Data Availability}

The data used to support the findings of this study are included within the article.

\section{Conflicts of Interest}

The authors declare that they have no conflicts of interest.

\section{Acknowledgments}

The study was supported by the Finance Science and Technology Project of Hainan Province (ZDYF 2019200) and the National Natural Science Foundation (81760310).

\section{References}

[1] F. Bray, J. Ferlay, I. Soerjomataram, R. L. Siegel, L. A. Torre, and A. Jemal, "Global cancer statistics 2018: GLOBOCAN estimates of incidence and mortality worldwide for 36 cancers in 185 countries," CA: a Cancer Journal for Clinicians, vol. 68, no. 6, pp. 394-424, 2018.

[2] W. Chen, R. Zheng, H. Zeng, and S. Zhang, "Epidemiology of lung cancer in China," Thoracic Cancer, vol. 6, no. 2, pp. 209215, 2015.

[3] P. C. Hoffman, A. M. Mauer, and E. E. Vokes, "Lung cancer," Lancet, vol. 355, no. 9202, pp. 479-485, 2000.

[4] S. Devarakonda, D. Morgensztern, and R. Govindan, "Genomic alterations in lung adenocarcinoma," Lancet Oncologia, vol. 16, no. 7, pp. e342-e351, 2015.

[5] Q. Y. Hong, G. M. Wu, G. S. Qian et al., "Prevention and management of lung cancer in China," Cancer, vol. 121, Suppl 17, pp. 3080-3088, 2015.

[6] M. T. Landi, Y. Zhao, M. Rotunno et al., "MicroRNA expression differentiates histology and predicts survival of lung cancer," Clinical Cancer Research, vol. 16, no. 2, pp. 430-441, 2010.

[7] J. S. Kim, E. J. Kim, S. Lee et al., "MiR-34a and miR-34b/c have distinct effects on the suppression of lung adenocarcinomas," Experimental \& Molecular Medicine, vol. 51, no. 1, pp. 1-10, 2019.

[8] J. Gu, G. Wang, H. Liu, and C. Xiong, "SATB2 targeted by methylated miR-34c-5p suppresses proliferation and metastasis attenuating the epithelial-mesenchymal transition in colorectal cancer," Cell Proliferation, vol. 51, no. 4, article e12455, 2018.

[9] M. Re, A. Ceka, C. Rubini et al., "MicroRNA-34c-5p is related to recurrence in laryngeal squamous cell carcinoma," Laryngoscope, vol. 125, no. 9, pp. E306-E312, 2015.

[10] A. Nabetani, T. Koujin, C. Tsutsumi, T. Haraguchi, and Y. Hiraoka, "A conserved protein, Nuf2, is implicated in connecting the centromere to the spindle during chromosome segregation: a link between the kinetochore function and the spindle checkpoint," Chromosoma, vol. 110, no. 5, pp. 322 334, 2001.

[11] W. Obara, F. Sato, K. Takeda et al., "Phase I clinical trial of cell division associated 1 (CDCA1) peptide vaccination for castration resistant prostate cancer," Cancer Science, vol. 108, no. 7, pp. 1452-1457, 2017.

[12] P. Hu, X. Chen, J. Sun, P. Bie, and L. D. Zhang, "siRNA-mediated knockdown against NUF2 suppresses pancreatic cancer proliferation in vitro and in vivo," Bioscience Reports, vol. 35, no. $1,2015$.

[13] Y. Kobayashi, A. Takano, Y. Miyagi et al., "Cell division cycleassociated protein 1 overexpression is essential for the malignant potential of colorectal cancers," International Journal of Oncology, vol. 44, no. 1, pp. 69-77, 2014.

[14] A. Warth, T. Muley, M. Meister et al., "The novel histologic International Association for the Study of Lung Cancer/American Thoracic Society/European Respiratory Society classification system of lung adenocarcinoma is a stage-independent predictor of survival," Journal of Clinical Oncology, vol. 30, no. 13, pp. 1438-1446, 2012.

[15] K. Inamura, "Diagnostic and therapeutic potential of microRNAs in lung cancer," Cancers, vol. 9, no. 12, p. 49, 2017.

[16] H. X. Gao, Y. Su, A. L. Zhang, J. W. Xu, Q. Fu, and L. Yan, "miR-34c-5p plays a protective role in chronic obstructive pulmonary disease via targetingCCL22," Experimental Lung Research, vol. 45, no. 1-2, pp. 1-12, 2019.

[17] F. Akbas, E. Coskunpinar, E. Aynaci, Y. M. Oltulu, and P. Yildiz, "Analysis of serum micro-RNAs as potential biomarker in chronic obstructive pulmonary disease," Experimental Lung Research, vol. 38, no. 6, pp. 286-294, 2012.

[18] J. Gandla, S. K. Lomada, J. Lu, R. Kuner, and K. K. Bali, “miR$34 c-5 p$ functions as pronociceptive microRNA in cancer pain by targeting Cav2.3 containing calcium channels," Pain, vol. 158, no. 9, pp. 1765-1779, 2017.

[19] Y. Wang, T. Lu, Y. Wo et al., "Identification of a putative competitive endogenous RNA network for lung adenocarcinoma using TCGA datasets," PeerJ, vol. 7, article e6809, 2019.

[20] C. Achari, S. Winslow, Y. Ceder, and C. Larsson, "Expression of miR-34c induces G2/M cell cycle arrest in breast cancer cells," BMC Cancer, vol. 14, no. 1, p. 538, 2014.

[21] G. Di Leva, M. Garofalo, and C. M. Croce, "MicroRNAs in cancer," Annual Review of Pathology, vol. 9, no. 1, pp. 287314, 2014.

[22] S. Catuogno, L. Cerchia, G. Romano, P. Pognonec, G. Condorelli, and V. de Franciscis, "miR-34c may protect lung cancer cells from paclitaxel-induced apoptosis," Oncogene, vol. 32, no. 3, pp. 341-351, 2013.

[23] Y. Wan, A. Shen, F. Qi et al., "Pien Tze Huang inhibits the proliferation of colorectal cancer cells by increasing the expression of miR-34c-5p," Experimental and Therapeutic Medicine, vol. 14, no. 4, pp. 3901-3907, 2017.

[24] S. Cordova-Rivas, I. Fraire-Soto, A. Mercado-Casas Torres et al., " $5 p$ and $3 p$ strands of miR-34 family members have differential effects in cell proliferation, migration, and invasion in cervical cancer cells," International Journal of Molecular Sciences, vol. 20, no. 3, p. 545, 2019.

[25] L. Hu, J. Ai, H. Long et al., "Integrative microRNA and gene profiling data analysis reveals novel biomarkers and mechanisms for lung cancer," Oncotarget, vol. 7, no. 8, pp. 84418454, 2016. 
[26] N. Li, D. Mao, Y. Cao, H. Li, F. Ren, and K. Li, "Downregulation of SIRT6 by miR-34c-5p is associated with poor prognosis and promotes colon cancer proliferation through inhibiting apoptosis via the JAK2/STAT3 signaling pathway," International Journal of Oncology, vol. 52, no. 5, pp. 1515-1527, 2018.

[27] T. Zhang, Y. Zhou, S. T. Qi et al., "Nuf2 is required for chromosome segregation during mouse oocyte meiotic maturation," Cell Cycle, vol. 14, no. 16, pp. 2701-2710, 2015.

[28] Y. Xing, J. Zhang, L. Lu et al., "Identification of hub genes of pneumocyte senescence induced by thoracic irradiation using weighted gene coexpression network analysis," Molecular Medicine Reports, vol. 13, no. 1, pp. 107-116, 2016.

[29] W. Xu, Y. Wang, Y. Wang, S. Lv, X. Xu, and X. Dong, "Screening of differentially expressed genes and identification of NUF2 as a prognostic marker in breast cancer," International Journal of Molecular Medicine, vol. 44, no. 2, pp. 390-404, 2019.

[30] Y. Wang, P. Y. Tan, Y. A. Handoko et al., "NUF2 is a valuable prognostic biomarker to predict early recurrence of hepatocellular carcinoma after surgical resection," International Journal of Cancer, vol. 145, no. 3, pp. 662-670, 2019.

[31] H. L. Fu and L. Shao, "Silencing of NUF2 inhibits proliferation of human osteosarcoma Saos-2 cells," European Review for Medical and Pharmacological Sciences, vol. 20, no. 6, pp. 1071-1079, 2016.

[32] W. Yuan, S. Xie, M. Wang et al., "Bioinformatic analysis of prognostic value of ZW10 interacting protein in lung cancer," Oncotargets and Therapy, vol. 11, pp. 1683-1695, 2018.

[33] D. Zhang, E. Liu, J. Kang, X. Yang, and H. Liu, “miR-3613-3p affects cell proliferation and cell cycle in hepatocellular carcinoma," Oncotarget, vol. 8, no. 54, pp. 93014-93028, 2017. 